\title{
Comparison of Three Different Noninvasive Ventilation Strategies as Initial Respiratory Support in Very Low Birth Weight Infants
}

\author{
Mehmet BUYUKTIRYAKI, Nilufer OKUR, Fatma Nur SARI, Buse BEKMEZ, Handan BEZIRGANOGLU, Ufuk CAKIR, \\ Evrim ALYAMAC DIZDAR, Serife Suna OGUZ \\ Neonatology, Zekai Tahir Burak Maternity Teaching Hospital, Ankara, Turkey.
}

Background: Nasal continuous positive airway pressure (NCPAP) has been widely used for the respiratory support of preterm infants in many neonatal intensive care units. However, studies using NCPAP as initial respiratory support in respiratory distress syndrome (RDS) have reported noninvasive ventilation (NIV) failure rates of $25-50 \%$ which has led to the use of alternative NIV strategies such as nasal intermittent positive pressure ventilation (NIPPV) or bi-level positive airway pressure (BiPAP).

Objective: To compare the failure of NIV and neonatal outcomes between NCPAP, BiPAP and NIPPV as the initial respiratory support with less invasive surfactant administration (LISA) in very low birth weight (VLBW) infants.

Methods: Medical records of 419 VLBW infants born at 26-30 weeks' gestation who did not require intubation in the delivery

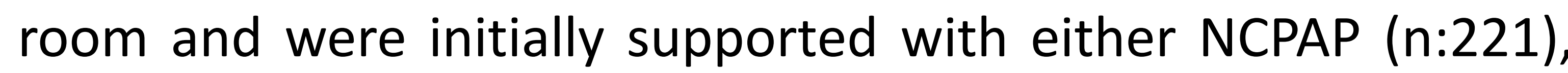
BiPAP (n:101), or NIPPV (n:97) were retrospectively reviewed. LISA approach was preferred in case of surfactant requirement. The primary outcome was the failure of NIV within the first 72 hours of life.

Results: Patient characteristics' of the groups were similar (Table 1). The failure of NIV within the first 72 hours of life was significantly higher in the NCPAP group (29.4\%) compared to the BiPAP $(12.9 \%)$ or NIPPV $(12.4 \%)$ group $(p<0.001)$ (Table2). However, BiPAP and NIPPV groups were similar in terms of NIV failure $(p=0.91)$. Multivariable logistic regression analysis showed that antenatal steroid administration (OR: $0.45,95 \% \mathrm{Cl}$ 0.26-0.80; $p<0.001$ ) and NCPAP support (OR: $3.08,95 \% \mathrm{Cl} 1.81-$ 5.22; $p<0.001$ ) were independent factors that influence failure of NIV within the first 72 hours of life. There was no difference between the groups in terms of preterm morbidity (Table 3 ).
TABLE 1. Characteristics of the patients

\begin{tabular}{lcccc}
\hline & $\begin{array}{c}\text { NCPAP } \\
(\mathbf{n}=\mathbf{2 2 1})\end{array}$ & $\begin{array}{c}\text { BiPAP } \\
(\mathbf{n}=101)\end{array}$ & $\begin{array}{c}\text { NIPPV } \\
(\mathbf{n}=97)\end{array}$ & $\mathbf{p}$ \\
\hline Birth weight $(\mathrm{g})^{*}$ & $1080 \pm 212$ & $1083 \pm 194$ & $1112 \pm 215$ & 0.43 \\
Gestational age (week)* & $28.1 \pm 1.1$ & $28.2 \pm 1.1$ & $28 \pm 1$ & 0.47 \\
SGA, $\mathrm{n}(\%)$ & $26(11.8)$ & $9(8.9)$ & $10(10.3)$ & 0.73 \\
Male gender, $\mathrm{n}(\%)$ & $101(45.7)$ & $51(50.5)$ & $54(55.7)$ & 0.25 \\
Caesarean delivery, $\mathrm{n}(\%)$ & $185(83.7)$ & $85(84.2)$ & $83(85.6)$ & 0.91 \\
Antenatal steroids, $\mathrm{n}(\%)$ & $172(77.8)$ & $79(78.2)$ & $77(79.4)$ & 0.95 \\
APGAR score at 5 min ${ }^{+}$ & $8(7-8)$ & $8(7-8)$ & $8(7-8)$ & 0.18 \\
Multiple births, $\mathrm{n}(\%)$ & $48(21.7)$ & $25(24.8)$ & $22(22.7)$ & 0.83 \\
Preeclampsia, $\mathrm{n}(\%)$ & $46(20.8)$ & $20(19.8)$ & $23(23.7)$ & 0.77 \\
Chorioamnionitis, $\mathrm{n}(\%)$ & $24(10.9)$ & $11(10.9)$ & $9(9.3)$ & 0.9 \\
\hline
\end{tabular}

${ }^{*}$ mean $\pm S D$ tmedian, interquartile range (IQR). SGA, Small for gestational age (below the $10^{\text {th }}$ centile)

TABLE 2. Primary and secondary outcomes in study groups

\begin{tabular}{|c|c|c|c|c|c|}
\hline & $\begin{array}{l}\text { NCPAP } \\
(n=221)\end{array}$ & $\begin{array}{c}\text { BiPAP } \\
(n=101)\end{array}$ & $\begin{array}{l}\text { NIPPV } \\
(n=97)\end{array}$ & $p$ & \\
\hline \multicolumn{6}{|l|}{ Primary outcomes } \\
\hline $\begin{array}{l}\text { NIV failure in the first } 72 \mathrm{~h} \text { of } \\
\text { life, } n(\%)\end{array}$ & $65(29.4)$ & $13(12.9)$ & $12(12.4)$ & $<0.001$ & $0.001^{\mathrm{a}, \mathrm{b}}$ \\
\hline \multicolumn{6}{|l|}{ Secondary outcomes } \\
\hline Required surfactant, $n(\%)$ & $130(58.8)$ & $42(41.6)$ & $40(41.2)$ & 0.002 & $0.004^{\mathrm{a}, \mathrm{b}}$ \\
\hline Overall rate of intubation, $n$ (\%) & $101(45.7)$ & $31(30.7)$ & $32(33)$ & 0.01 & $0.01^{\mathrm{a}}, 0.03^{\mathrm{b}}$ \\
\hline Duration of MV, days* & $0(0-3)$ & $0(0-2.5)$ & $0(0-2)$ & 0.17 & \\
\hline Duration of NIV, days* & $5(2-12)$ & $4(2-8)$ & $4(2-7)$ & 0.09 & \\
\hline $\begin{array}{l}\text { Duration of supplemental } \\
\text { oxygen, days* }\end{array}$ & $10(4-22)$ & $6(3-13.5)$ & $5(2-11)$ & $<0.001$ & $\begin{array}{l}0.002^{\mathrm{a}} \\
<0.001^{\mathrm{b}}\end{array}$ \\
\hline Pneumothorax, n (\%) & $6(2.7)$ & $3(3)$ & $3(3.1)$ & 0.97 & \\
\hline BPD, n (\%) & $29(13.1)$ & $9(8.9)$ & $8(8.2)$ & 0.32 & \\
\hline $\begin{array}{l}\text { Survival without BPD at } 36 \\
\text { weeks PMA, } n(\%)\end{array}$ & $172(77.8)$ & $84(83.2)$ & $79(81.4)$ & 0.49 & \\
\hline
\end{tabular}

${ }^{*}$ median, interquartile range (IQR). BPD, Bronchopulmonary dysplasia; NIV, Non-invasive ventilation; PMA, Postmenstrual age. p: Comparison of all 3 groups; pa: Comparison of NCPAP with BiPAP; pb: Comparison of NCPAP with NIPPV

TABLE 3. Preterm morbidities of study groups

\begin{tabular}{l|cccc}
\hline & $\begin{array}{c}\text { NCPAP } \\
(\mathbf{n}=\mathbf{2 2 1})\end{array}$ & $\begin{array}{c}\text { BiPAP } \\
(\mathbf{n}=\mathbf{1 0 1 )}\end{array}$ & $\begin{array}{c}\text { NIPPV } \\
(\mathbf{n}=\mathbf{9 7})\end{array}$ & $\mathbf{p}$ \\
\hline hsPDA, $\mathrm{n}(\%)$ & $66(29.9)$ & $31(30.7)$ & $28(28.9)$ & 0.96 \\
IVH> grade II, $\mathrm{n}(\%)$ & $13(5.9)$ & $4(4)$ & $5(5.2)$ & 0.77 \\
Periventricular leukomalacia, $\mathrm{n}(\%)$ & $11(5.1)$ & $3(3)$ & $4(4.2)$ & 0.70 \\
Spontaneous intestinal perforation, $\mathrm{n}(\%)$ & $4(1.8)$ & $1(1)$ & $1(1)$ & 0.78 \\
NEC $\geq$ stage II, $\mathrm{n}$ (\%) & $4(1.8)$ & - & $2(2.1)$ & 0.37 \\
ROP that required laser treatment, $\mathrm{n}(\%)$ & $18(9)$ & $6(6.8)$ & $5(5.3)$ & 0.51 \\
Duration of hospitalisation, days* & $65 \pm 22.8$ & $57.4 \pm 23$ & $54.4 \pm 22.9$ & $<0.001^{*}$ \\
\hline
\end{tabular}

NEC, Necrotising enterocolitis; ROP, Retinopathy of prematurity.

Conclusions: NIPPV or BiPAP as the initial respiratory support with LISA approach reduced the need for invasive ventilation in infants born at 26-30 weeks' gestation.

\section{References:}

1. Roberts CT, Davis PG, Owen LS. Neonatal non-invasive respiratory support: Synchronised NIPPV, nonsynchronised NIPPV or bi-level CPAP: What is the evidence in 2013. Neonatology 2013;104:203-9.

Salvo V, Lista G, Lupo E, et al. Comparison of three non-invasive ventilation strategies (NSIPPV/BiPAP/NCPAP) for RDS in VLBW infants. J Matern Fetal Neonatal Med 2017:1-7.

3. Salvo V, Lista G, Lupo E, et al. Noninvasive ventilation strategies for early treatment of RDS in preterm infants: an RCT. Pediatrics 2015;135:444-51. 